\title{
Decaying Warm Dark Matter and Neutrino Masses
}

\author{
M. Lattanzi ${ }^{1, *}$ and J. W. F. Valle ${ }^{2, \dagger}$ \\ ${ }^{1}$ Oxford Astrophysics, Denis Wilkinson Building, Keble Road, OX1 3RH, Oxford, United Kingdom \\ ${ }^{2}$ Instituto de Física Corpuscular-C.S.I.C./Universitat de València Campus de Paterna, Apt 22085, E-46071 València, Spain
}

(Received 27 May 2007; published 20 September 2007)

\begin{abstract}
Neutrino masses may arise from spontaneous breaking of ungauged lepton number. Because of quantum gravity effects the associated Goldstone boson-the majoron-will pick up a mass. We determine the lifetime and mass required by cosmic microwave background observations so that the massive majoron provides the observed dark matter of the Universe. The majoron decaying dark matter scenario fits nicely in models where neutrino masses arise via the seesaw mechanism, and may lead to other possible cosmological implications.
\end{abstract}

DOI: 10.1103/PhysRevLett.99.121301

A long-standing challenge in particle cosmology is to elucidate the nature of dark matter and its origin. $\mathrm{A} \mathrm{keV}$ weakly interacting particle could provide a sizeable fraction of the critical density $\rho_{\text {cr }}=1.88 \times 10^{-29} \mathrm{~h}^{2} \mathrm{~g} / \mathrm{cm}^{3}$ and possibly play an important role in structure formation, since the associated Jeans mass lies in the relevant range [1]. Although we now know from neutrino oscillation experiments that neutrinos do have mass [2], recent cosmological data [3] as well as searches for distortions in $\beta$ [4] and double $\beta$ decay spectra [5] place a stringent limit on the absolute scale of neutrino mass that precludes neutrinos from being viable warm dark matter candidates [6] and from playing a direct role in structure formation.

If neutrino masses arise from the spontaneous violation of ungauged lepton number there must exist a pseudoscalar gauge singlet Nambu-Goldstone boson, the majoron $[7,8]$. This may pick up a mass from nonperturbative gravitational effects that explicitly break global symmetries [9]. Despite the fact that the majorons produced at the corresponding spontaneous $L$-violation phase will decay, mainly to neutrinos, they could still provide a sizeable fraction of the dark matter in the Universe since its couplings are rather tiny.

The decaying warm dark matter particle idea is not new in itself. However, since early attempts [10], there have been important observational developments which must be taken into account in order to assess its viability. Specially relevant are the recent cosmological microwave observations from the Wilkinson Microwave Anisotropy Probe (WMAP) [11]. For definiteness here we adopt the very popular possibility that neutrino masses arise a la seesaw [12].

In the following we consider the majoron decaying dark matter (DDM) idea in a modified $\Lambda$ CDM cosmological model in which the dark matter particle is identified with the weakly interacting majoron $J$ with mass in the $\mathrm{keV}$ range. The majoron is not stable but decays nonradiatively with a small decay rate $\Gamma$. In this DDM scenario, the anisotropies of the cosmic microwave background (CMB) can be used to constrain the lifetime $\tau=\Gamma^{-1}$
PACS numbers: 95.35.+d, 14.60.Pq, 14.80.Mz, 98.70.Vc

and the present abundance $\Omega_{J}$ of the majoron; here we show that the cosmological constraints on DDM majorons not only can be fulfilled but also can easily fit into a comprehensive global picture for neutrino mass generation with spontaneous violation of lepton number.

Although majorons could result from a phase transition, we first consider them to be produced thermally, in equilibrium with photons in the early Universe. In this case the majoron abundance $n_{J}$ at the present time $t_{0}$ will be, owing to entropy conservation and taking into account their finite lifetime:

$$
\frac{n_{J}\left(t_{0}\right)}{n_{\gamma}\left(t_{0}\right)}=\frac{43 / 11}{N_{D}} \frac{n_{J}\left(t_{D}\right)}{n_{\gamma}\left(t_{D}\right)} e^{-t_{0} / \tau}
$$

where $t_{D}$ is the time of majoron decoupling, and $N_{D}$ denotes the number of quantum degrees of freedom at that time. If $T\left(t_{D}\right) \geqslant 170 \mathrm{GeV}$, then $N_{D}=427 / 4$ for the particle content of the standard model. On the other hand, in the context of a supersymmetric extension of the standard model of particle physics, there would possibly be, at sufficiently early times, about twice that number of degrees of freedom. Moreover, just after decoupling, the majoron to photon ratio $r \equiv n_{J}\left(t_{D}\right) / n_{\gamma}\left(t_{D}\right)$ is equal to $1 / 2$. The present density parameter of majorons is then

$$
\Omega_{J} h^{2}=\frac{m_{J}}{1.25 \mathrm{keV}} e^{-t_{0} / \tau}
$$

where we have used the standard value of $N_{D}$.

Alternatively, if majorons were produced already out of equilibrium there is a range of possible models, which we write generically as

$$
\Omega_{J} h^{2}=\beta \frac{m_{J}}{1.25 \mathrm{keV}} e^{-t_{0} / \tau},
$$

where the quantity $\beta$ parametrizes our ignorance about both the exact production mechanism, and the exact value of $N_{D}$. When $\beta=1$, we recover the scenario described above, with $r=1 / 2$ and $N_{D}=427 / 4$.

Clearly, if the majoron is to survive as a dark matter particle it must be long-lived, $\tau_{J} \geq t_{0}$. However, a more 
stringent bound follows by studying the effect of a finite majoron lifetime on the cosmological evolution and, in particular, on the CMB anisotropy spectrum. In the DDM scenario, due to particle decays, the dark matter density is decreasing faster than in the standard cosmological picture. This changes the time $t_{\mathrm{eq}}$ of radiation-matter equality. This means that, for a fixed $\Omega_{J}$, there will be more dark matter at early times, and the equality will take place earlier, as illustrated in Fig. 1. The present amount of dark matter is $\Omega_{\mathrm{DM}}=0.25$ for both models; $\Gamma^{-1}=14 \mathrm{Gyr}$ in the DDM model. Other relevant parameters are $\omega_{b}=2.23 \times 10^{-2}$ and $h=0.7$. The time at which the blue and red lines cross is the time of matter-radiation equality; for fixed $\Omega_{\mathrm{DM}}$, it shifts to earlier times as the majoron lifetime decreases.

The time of matter-radiation equality has a direct effect on the CMB power spectrum. The gravitational potentials are decaying during the radiation dominated era; this means that photons will receive an energy boost after crossing potential wells. This so-called early integrated Sachs-Wolfe effect ceases when matter comes to dominate the Universe, since the potential is constant during matter domination. The overall effect is to increase the power around the first peak as the equality moves to later times.

On the other hand, since $\tau \gtrsim t_{0}$, we expect the majoron decays to make the gravitational potentials vary again in the late stage of the cosmological evolution. This will induce a similar effect to the one described above, only affecting larger scales due to the increased horizon size. This late integrated Sachs-Wolfe effect results then in an excess of power at small multipoles.

Both effects can be used in principle to constrain the majoron lifetime and cosmological abundance. In order to be quantitative, we have developed a modified version of the CAMB code [13], which enables us to compute the CMB

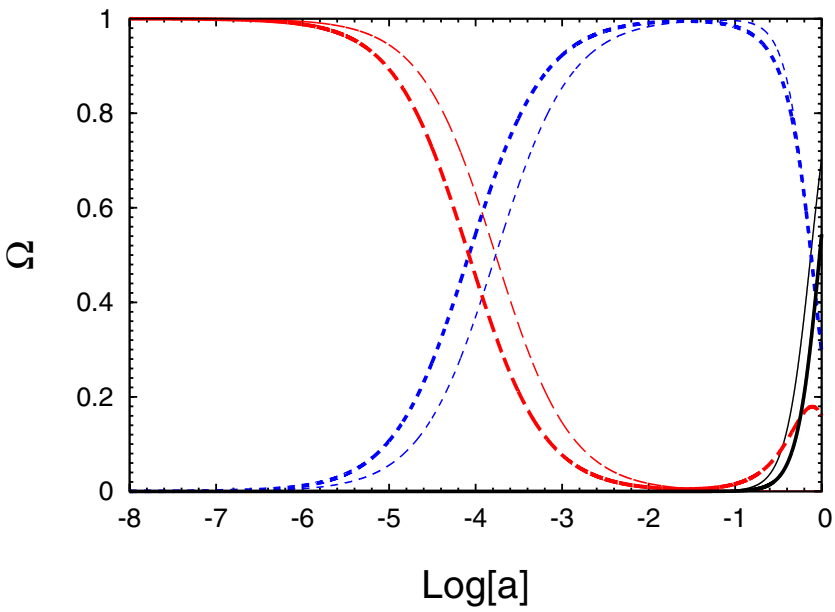

FIG. 1 (color online). Evolution of abundances in the standard (thin lines) and DDM (thick lines) universe scenario: blue (short dashed line), red (long dashed line) and black (solid line) correspond to the matter, radiation, and $\Lambda$ components, respectively. anisotropy spectrum once the majoron lifetime and abundance are given in addition to the standard $\Lambda \mathrm{CDM}$ model parameters.

Even if a $\mathrm{keV}$ majoron would constitute a warm dark matter particle, it behaves as cold dark matter insofar as the calculation of its effect on the CMB spectrum is concerned, since $\mathrm{CMB}$ measurements cannot discriminate between cold and warm dark matter [14]. The latter behaves differently from a cold one on scales smaller than its freestreaming length $\lambda_{\mathrm{fs}}$. For a particle mass in the $\mathrm{keV}$ range, we have $\lambda_{f s} \sim 1 \mathrm{Mpc}$, which corresponds in the CMB to a multipole $\ell \sim$ a few thousands. The formalism needed to account for the cosmological evolution of an unstable relic and of its light decay products, has been developed, for example, in Refs. [15,16], including the modifications in both background quantities and perturbation evolution.

Two distinct mechanisms effective at very different times characterize the effect of DDM on the CMB. It is therefore convenient to choose a parametrization that can take advantage of this fact. In particular, the "natural" parametrization $\left(\Omega_{J}, \Gamma\right)$ has the drawback that both parameters affect the time of matter-radiation equality. It is more convenient to define the quantity

$$
\left.Y \equiv \frac{\rho_{J}}{\rho_{b}}\right|_{t=t_{\text {early }}},
$$

where $\rho_{b}$ is the energy density of baryons, and $t_{\text {early }} \ll$ $t_{0} \lesssim \tau$. As long as this condition is fulfilled, the value of $Y$ does not depend on the particular choice of $t_{\text {early }}$, since the ratio $\rho_{J} / \rho_{b}$ is asymptotically constant at small times. Given that $t_{\text {eq }} \ll \tau$ we can use the value of $Y$ to parametrize the relative abundance of majorons at matter-radiation equality. In order to simplify the notation let us also define $\Gamma_{18} \equiv \Gamma /\left(10^{-18} \mathrm{sec}^{-1}\right)$; in this way, $\Gamma_{18}=1$ corresponds to a lifetime $\tau \simeq 30 \mathrm{Gyr}$.

In the parametrization $(Y, \Gamma)$, fixing the other parameters, $Y$ determines the time of equality, while $\Gamma$ mainly affects the magnitude of the LISW effect. We show in Fig. 2 how the two physical effects are nicely separated in this parametrization. We start from a fiducial model with $\Gamma_{18}=0$ and $Y=4.7$; all other parameters are fixed to their WMAP best-fit values. The values of $\Gamma_{18}$ and $Y$ are chosen in such a way to give $\Omega_{J} h^{2}=0.10$, so that this fiducial model reproduces exactly the WMAP best fit. At a larger majoron decay rate $\Gamma_{18}=1.2$, i.e., $\Gamma^{-1} \simeq 27 \mathrm{Gyr}$ the LISW effect makes, as expected, the power at small multipoles increase, while the shape of the spectrum around the first peak does not change, since the abundance of matter at early times has not changed. Finally, increasing $Y$ by $20 \%$ the height of the first peak decreases accordingly, while the largest angular scales (small $\ell$ ) are nearly unaffected. A small decrease in power in this region is actually observed, and can be explained by noticing that increasing the matter content we delay the onset of the $\Lambda$ dominated era, reducing the $\Lambda$ contribution to the LISW effect. 
Another advantage of using the above parametrization is that $Y$ is directly related to the majoron mass through

$$
Y=0.71\left(\frac{m_{J}}{\mathrm{keV}}\right)\left(\frac{\beta}{\Omega_{b} h^{2}}\right)
$$

We are now ready to compute the constraints that CMB observations put on the majoron abundance and lifetime. As seen from Fig. 2, even a lifetime twice as long as the present age of the Universe is quite at variance with respect to the WMAP data. However, one must take into account the fact the values of the other cosmological parameters can be arranged in such a way as to reduce or even cancel the conflict with observation, i.e., degeneracies may be present in parameter space. In order to obtain reliable constraints for the majoron mass and lifetime, we perform a statistical analysis allowing for the variation of all parameters. This is better accomplished using a Markov chain Monte Carlo approach; we used to this purpose the widely known COSMOMC code [17].

In our modified flat $(\Omega=1) \Lambda$ CDM model, all the dark matter is composed of majorons. This means that no stable cold dark matter is present [18]. The seven-dimensional parameter space we explore therefore includes the two parameters $(Y, \Gamma)$ defined above, in addition to the five standard parameters, namely: the baryon density $\Omega_{b} h^{2}$, the dimensionless Hubble constant $h$, the reionization optical depth $\tau$, the amplitude $A_{s}$ and spectral index $n_{s}$ of the primordial density fluctuations. The cosmological constant density $\Omega_{\Lambda}$ depends on the values of the other parameters due to the flatness condition. We compare our results with the CMB anisotropies observed by the WMAP experiment. Once the full probability distribution function for the seven base parameters has been obtained in this way, the proba-

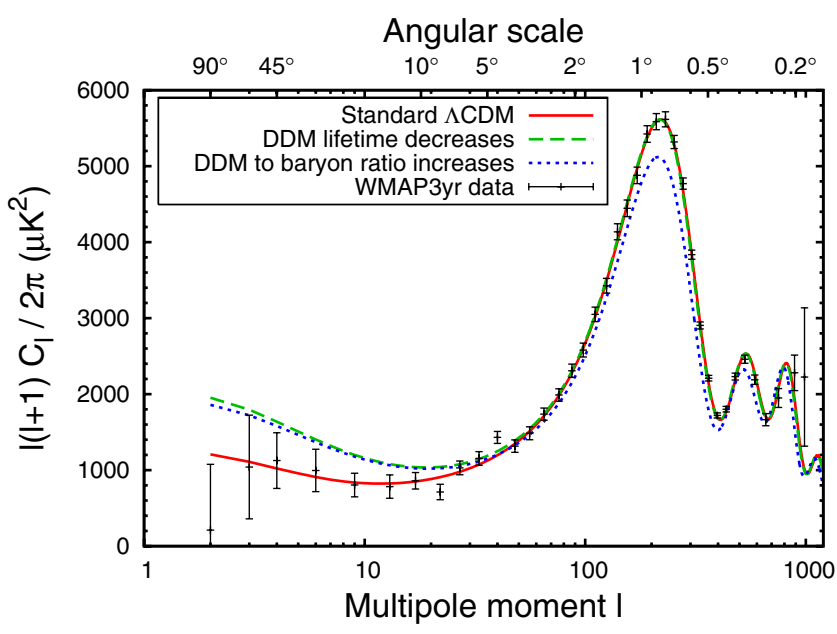

FIG. 2 (color online). Effect of DDM parameters on the CMB anisotropy spectrum. The value of the parameters are as follows. Red (solid) line: fiducial model $\left(\Gamma_{18}, Y\right)=(0,4.7)$. Green (dashed) line: $\left(\Gamma_{18}, Y\right)=(1.2,4.7)$. Blue (dotted) line: $\left(\Gamma_{18}, Y\right)=$ $(1.2,5.6)$. See text. bility densities for derived parameters, such as the majoron mass $m_{J}$, is obtained.

We show our result in Fig. 3, where we give the $68 \%$ and 95\% confidence contours in the $\left(m_{J}, \Gamma\right)$ plane, for the case $\beta=1$, i.e., thermal majoron production and $N_{D}=427 / 4$. We note that these parameters are not degenerate one with the other, so the respective constraints are independent. Similarly, we find no degeneracy between $\Gamma_{J}$ and the five standard parameters. The marginalized one-dimensional limits for $\Gamma_{J}$ and $m_{J}$ are

$$
\begin{gathered}
\Gamma_{J}<1.3 \times 10^{-19} \mathrm{sec}^{-1}, \\
0.12 \mathrm{keV}<m_{J}<0.17 \mathrm{keV} .
\end{gathered}
$$

Expressed in terms of the majoron lifetime our result implies $\tau>250 \mathrm{Gyr}$, nearly a factor 20 improvement with respect to the naive limit $\tau>t_{0} \simeq 14 \mathrm{Gyr}$, illustrating the power of CMB observations in constraining particle physics scenarios. Let us comment on the possibility that $\beta \neq 1$. From Eq. (3), it can be seen that this amounts to the transformation $m_{J} \rightarrow \beta m_{J}$. For example, as we have already pointed out, $N_{D}$ can be as large as $427 / 2$, so that the above limit would read $0.24 \mathrm{keV}<m_{J}<0.34 \mathrm{keV}$. In general, if we allow for the possibility of extra degrees of freedom in the early Universe, we always have $\beta<1$ and then $m_{J}>0.12 \mathrm{keV}$. If instead majorons are produced nonthermally, one will in general have $\beta>1$.

Let us now briefly comment on the particle physics model. The simplest possibility is that neutrino masses arise a la seesaw [12]. In the basis $\nu, \nu^{c}$ (where $\nu$ denote ordinary neutrinos, while $\nu^{c}$ are the $\mathrm{SU}(2) \otimes \mathrm{U}(1)$ singlet

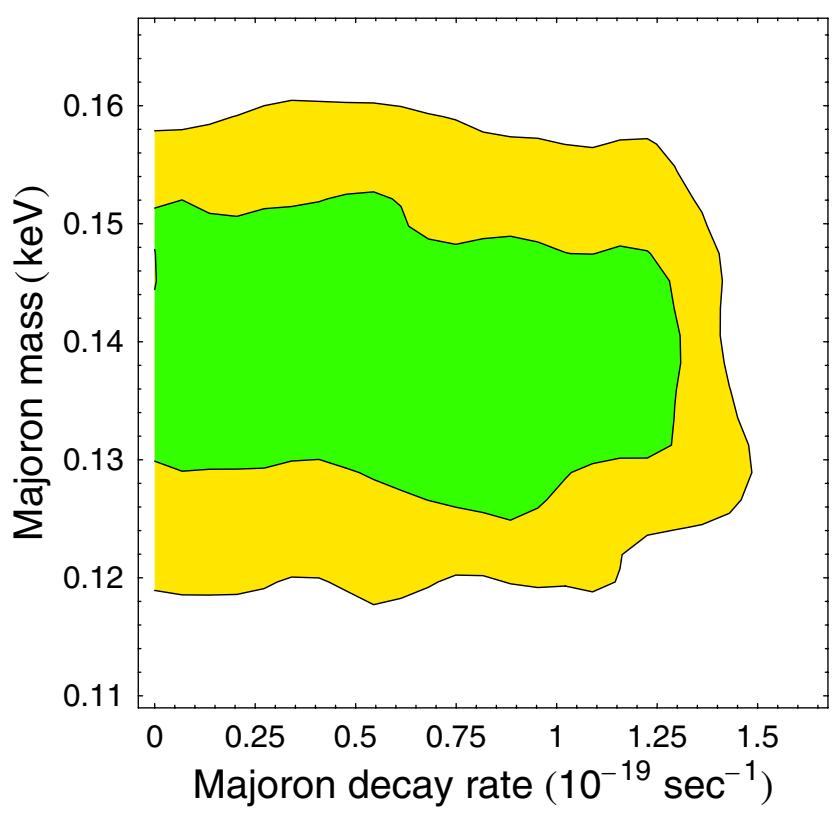

FIG. 3 (color online). Contours of the 68\% [green (dark) area] and $95 \%$ [yellow (light) area] confidence regions in the $\left(\Gamma_{J}, m_{J}\right)$ plane. 
"right-handed" neutrinos) the full neutrino mass matrix is given as

$$
\mathcal{M}_{\nu}=\left(\begin{array}{cc}
Y_{3} v_{3} & Y_{\nu} v_{2} \\
Y_{\nu}^{T} v_{2} & Y_{1} v_{1}
\end{array}\right)
$$

and involves, in addition to the singlet, also a Higgs triplet contribution [19] whose vacuum expectation value obeys a "vev" seesaw relation of the type $v_{3} v_{1} \sim v_{2}^{2}$. The Higgs potential combines spontaneous breaking of the lepton number and of the electroweak symmetry. The properties of the seesaw majoron and its couplings follow from the symmetry properties of the potential and were extensively discussed in [8]. Here we assume, in addition, that quantum gravity effects [9] produce nonrenormalizable Planckmass suppressed terms which explicitly break the global lepton number symmetry and provide the majoron mass, which we cannot reliably compute, but we assume that it lies in the cosmologically interesting $\mathrm{keV}$ range.

In all of such models the majoron interacts mainly with neutrinos, proportionally to their mass [8], leading to

$$
\tau(J \rightarrow \nu \nu) \approx \frac{16 \pi}{m_{J}} \frac{v_{1}^{2}}{m_{\nu}^{2}} .
$$

The limits obtained above from the WMAP data can be used to roughly constrain the lepton number breaking scale as $v_{1}^{2} \gtrsim 3 \times\left(10^{6} \mathrm{GeV}\right)^{2}$, for $m_{\nu} \simeq 1 \mathrm{eV}$.

The massive majoron has also a subleading radiative decay mode, $J \rightarrow \gamma \gamma$, making our DDM scenario potentially testable through studies of the diffuse photon spectrum in the far ultraviolet. A more extended investigation of these schemes will be presented elsewhere including other cosmological data such as the large scale structure data from the Sloan Digital Sky Survey [20]. In contrast, we do not expect the data from upcoming CMB experiments like Planck to substantially improve our bounds on the majoron decay rate, since they mainly affect the large angular scales where the error bars have already reached the limit given by cosmic variance. We also note that direct detection of a $\mathrm{keV}$ majoron is possible in a suitable underground experiment [21].

Work supported by MEC Grant No. FPA2005-01269, by EC Contracts RTN network No. MRTN-CT-2004-503369. M. L. is currently supported by INFN and the University of Rome "La Sapienza". The authors would like to acknowledge S. Pastor for computing help.

\footnotetext{
*mxl@astro.ox.ac.uk

†valle@ific.uv.es

http://ahep.uv.es
}

[1] J. R. Bond, G. Efstathiou, and J. Silk, Phys. Rev. Lett. 45, 1980 (1980); J. R. Bond, A. S. Szalay, and M. S. Turner, Phys. Rev. Lett. 48, 1636 (1982); H. Pagels and J. R. Primack, Phys. Rev. Lett. 48, 223 (1982); H. Yuksel, J.F. Beacom, and C.R. Watson, arXiv:0706.4084; U. Seljak, A. Makarov, P. McDonald, and H. Trac, Phys. Rev. Lett. 97, 191303 (2006); M. Viel, J. Lesgourgues, M. G. Haehnelt, S. Matarrese, and A. Riotto, Phys. Rev. Lett. 97, 071301 (2006).

[2] For an updated review see the arXiv version of $\mathrm{M}$. Maltoni, T. Schwetz, M. A. Tortola, and J. W. F. Valle, New J. Phys. 6, 122 (2004).

[3] S. Hannestad, Annu. Rev. Nucl. Part. Sci. 56, 137 (2006); J. Lesgourgues and S. Pastor, Phys. Rep. 429, 307 (2006).

[4] G. Drexlin (KATRIN Collaboration), Nucl. Phys. B, Proc. Suppl. 145, 263 (2005).

[5] H. V. Klapdor-Kleingrothaus, I. V. Krivosheina, A. Dietz, and O. Chkvorets, Phys. Lett. B 586, 198 (2004).

[6] G. Gelmini, D. N. Schramm, and J. W. F. Valle, Phys. Lett. B 146, 311 (1984); A. Doroshkevich, M. Khlopov, and A. A. Klypin, Mon. Not. R. Astron. Soc. 239, 923 (1989).

[7] Y. Chikashige, R. N. Mohapatra, and R. D. Peccei, Phys. Lett. B 98, 265 (1981).

[8] J. Schechter and J.W.F. Valle, Phys. Rev. D 25, 774 (1982).

[9] S. R. Coleman, Nucl. Phys. B310, 643 (1988); S. B. Giddings and A. Strominger, Nucl. Phys. B307, 854 (1988); E. K. Akhmedov, Z.G. Berezhiani, R. N. Mohapatra, and G. Senjanovic, Phys. Lett. B 299, 90 (1993).

[10] V. Berezinsky and J. W. F. Valle, Phys. Lett. B 318, 360 (1993).

[11] D. N. Spergel et al. (WMAP team), Astrophys. J. Suppl. Ser. 170, 377 (2007).

[12] For a recent review see J. W. F. Valle, J. Phys. Conf. Ser. 53, 473 (2006), based on lectures at the Corfu Summer Institute on Elementary Particle Physics in September 2005.

[13] A. Lewis, A. Challinor, and A. Lasenby, Astrophys. J. 538, 473 (2000).

[14] M. Viel, J. Lesgourgues, M. G. Haehnelt, S. Matarrese, and A. Riotto, Phys. Rev. D 71, 063534 (2005).

[15] M. Kaplinghat, R.E. Lopez, S. Dodelson, and R. J. Scherrer, Phys. Rev. D 60, 123508 (1999).

[16] K. Ichiki, M. Oguri, and K. Takahashi, Phys. Rev. Lett. 93, 071302 (2004).

[17] A. Lewis and S. Bridle, Phys. Rev. D 66, 103511 (2002).

[18] This happens, e.g., in models where supersymmetry with broken $R$ parity is the origin of neutrino mass; M. Hirsch and J. W. F. Valle, New J. Phys. 6, 76 (2004); M. Hirsch et al., Phys. Rev. D 62, 113008 (2000); 65 119901(E), 2002.

[19] J. Schechter and J. W.F. Valle, Phys. Rev. D 22, 2227 (1980).

[20] M. Lattanzi and J. W. F. Valle (to be published).

[21] R. Bernabei et al., Int. J. Mod. Phys. A 21, 1445 (2006). 